\title{
Definition of Maintenance Policies in Power Systems Using a Sequential Monte Carlo
}

Subject Headings. Electrical engineering, Maintenance, Planning.

Author Keywords. Failure rate, Maintenance, Monte Carlo, Reliability.

\section{Introduction}

Monte Carlo simulations remain the standard method to compute estimates of reliability indices in Power Systems. These simulations are divided in two approaches: the chronological and the non chronological. One of the goals of this paper is the inclusion of different types of maintenance policies in the life cycle of a Power System. Sometimes, maintenances are based on the elapse of time and, for this reason, this paper presents a chronological simulation. However, most of reliability studies that present a chronological approach use an exponential distribution to generate the life cycle of the components of a Power System. The use of this approach simplifies a lot the generation of the life cycles. The problem of this approach lies on the consequence of the use of an exponential distribution. To use such distribution, the failure rate $\lambda$ of the components need to be constant. In Power Systems, this assumption is not true. The failure rate $\lambda$ of electrical components suffer an evolution during its life. The well-known bathtub curve illustrates this evolution. Therefore, this paper presents a new approach that allows to include the bathtub curve in order to achieve a realistic situation in the Power Systems reliability evaluations. By introducing, in this paper, the existence of a variable failure rate, the process of degradation of the components of a power system is also included. Therefore, another very important aspect in the power systems reliability evaluation is treated: the maintenance policies. This paper presents three different Monte Carlo applications that correspond to three different maintenance policies: reactive maintenance, preventive maintenance and predictive maintenance. The inclusion of this new aspect has one goal: the improvement of the reliability indices through the extension of the useful life period of the components by applying different maintenance strategies.

\section{The Monte Carlo Methods}

Several techniques were developed in order to evaluate the power systems reliability. The two main approaches that are described in the literature are: the deterministic approach 
(Miranda, 2005) and the probabilistic approach (Billiton and Allan 1996). It is now known that the systems behavior is stochastic and, for that reason, the evaluation of such systems should be made by probabilistic techniques. Analytical (Billiton and Allan 1996) and simulation (Miranda, 2005), (Billinton and Li 1994) are the two different methods that compose the probabilistic approach. Simulation methods are based on Monte Carlo simulations (Billinton and Li 1994), (Miranda, 2005). The simulation techniques, often known as Monte Carlo simulations, estimate the reliability indices using a random sampling of scenarios. The Monte Carlo simulation can take basically two major types: chronological/sequential simulation and not chronological simulation.

The non chronological approach simulates the basic intervals of the system lifetime by choosing intervals randomly. Therefore, there is no place to consider operating and failure times, but only probabilities to find the equipments in failure mode. On the other hand, the sequential approach simulates the basic intervals in chronological order.

Chronological simulation is meant to represent the course of life of the electrical system. Therefore, not only it simulates the times until each equipment fail but also the times of their repair. In chronological models, the simulation follows the line of temporal development and therefore we can use the metaphor of the film in opposition to a collection of photos. Therefore, the life cycle of a power system can be obtained through the combination of the life cycles of each component (Sankarakrishnan and Billinton 1995). In order to develop a chronological simulation is not enough to know the unavailability (probability of failure) of the components of the system, the so called Forced Outage Rate (F.O.R). It's necessary, for each of them, to know the failure density function. This function is associated with the operating and failure (repair) times. The exponential function is the most used to model these times. In this paper, a new approach to calculate the operating times will be used (Billinton and Allan 1983). This will add some news to the actual state of the art. Considering the above points, the great advantage of the sequential Monte Carlo is the possibility to include chronological issues. Usually, the process is based on a uniform distribution. There are two particular features which special attention should be given in this work. First, the failure rate $\lambda$ of the components will not be considered constant. Therefore, it is intended to simulate the existence of the bathtub curve that is composed for three phases: childhood, useful life and the wear-out period. These three different stages occur sequentially according to the elapse of the components life time. Another important goal of this work is the implementation of different types of maintenance to a power system. It is known that the maintenance of a given component occurs in specific periods of its life. Therefore, the reasons presented above explain why the Monte Carlo method developed was based on a chronological simulation. The general scheme of a typical chronological Monte Carlo process will now be presented:

1. Simulate the operating times for each component of the system, according to the probability distribution used. This constitutes the initial state of the system;

2. Identify the lowest simulated time. The corresponding component will be called F;

3. Update the system load for this particular moment;

4. State evaluation. According to the current state of each component, the load curtailment is calculated;

5. Update the reliability indices accumulators;

6. Simulate a new operating or repair time to the component $F$. The type of simulated time depends on the previous state of the component $F$. Therefore, if component $F$ 
was in operational mode, this means that this component is the next to fail. Thus, a repair time will be generated;

7. Update the components $F$ state and its lifetime;

8. Evaluate the lifetime of the system. Tests if the system has already completed one year and make the necessary updates, including the update of the coefficient of variation $\beta$;

9. Evaluate the coefficient of variation $\beta$. If convergence has not been reached, return to step 2. Otherwise, proceed to calculate the expected values and distributions of reliability indices and finish the process.

In sequential Monte Carlo, a life cycle is simulated for each component of the system. Having this step done, it is possible to check, at each time, if there is load curtailment or not. Then, the reliability indices can be calculated.

\section{The Maintenance Strategies}

Maintenance practices are an essential step of the planning, construction and operation of a power system. Looking at the maintenance policies that, currently, are presented by the companies, it's possible to say that most of them see these procedures as actions associated with the failure of equipment. However, these practices should be taken in order to prevent a component from failing or to repair normal equipment degradation. Unfortunately, data obtained in many studies prove that the main concern of companies is their economic requirements, while also adhering to the constraints set by system and customer requirements. This fact means that companies prefer to wait until a component failure and, only after this, they take the necessary measures to replace the normal operation.

The operation of a component is a time based-function. The shape of the hazard rate curve is often referred as a bathtub curve. As it was already stated, this curve can be divided into three different regions. Region 1 is characterized by the decrease of the hazard rate as function of time or age. The high failure rate associated with this region is linked to manufacturing errors or improper design. This region is followed by a nearly constant failure rate and is known as the useful life period or normal operating phase. In this region failures occur purely by chance and this is the only region in which the exponential distribution is valid. The third region represents the wear-out or fatigue phase and it is characterized by a rapidly increasing of the hazard rate with time. These three regions can also be identified in figure 1.

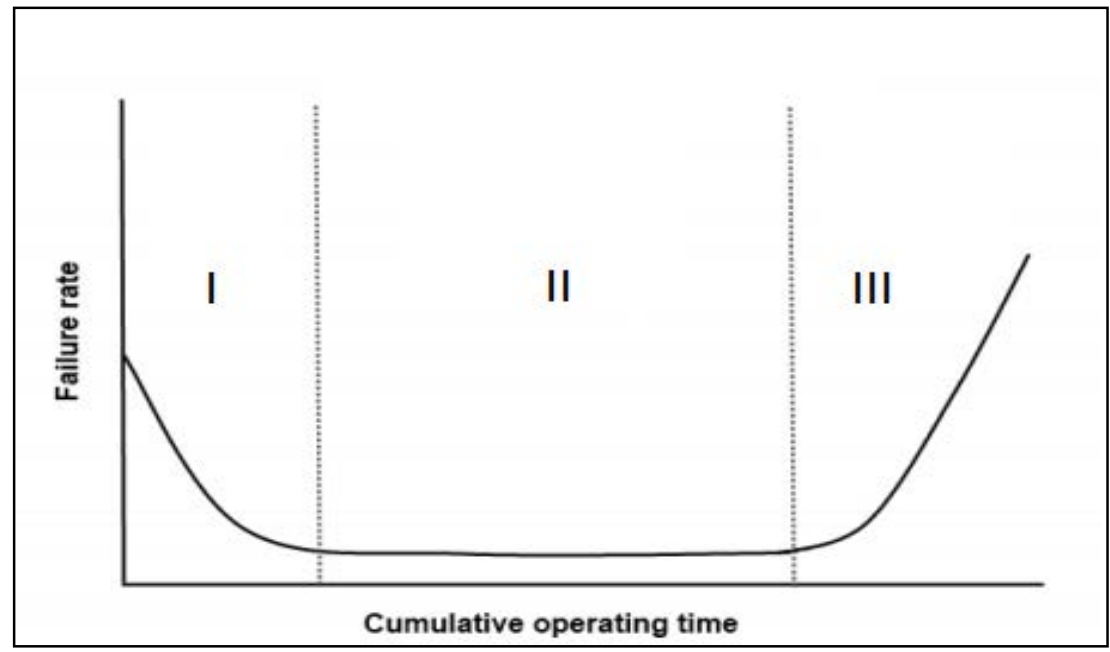

Figure 1: Typical electronic component failure rate $\lambda$ as a function of age (Billinton and Allan 1983)

The three different maintenance strategies that will be addressed in this paper will be now explained. 


\subsection{Reactive Maintenance}

Reactive Maintenance procedure is basically a mode in which maintenance actions are not taken. In this method, there are no concerns to keep or to extend the originally designed lifetime of the components. Some studies indicate that this type of maintenance is still predominant (Sullivan et al.). It is difficult to find advantages in this kind of maintenance. It can be seen as double-edge sword. On the one hand, the main thought of the companies' plan of strategy is: "if we set apart the maintenance program, we are saving money until some component has a failure. Furthermore, we increase the budget that can be spent in other fields." That can be seen has an advantage, but the problem lies on the other edge of the sword. When the companies set apart the maintenance, they forget that they are shortening the life of the equipment. This, actually, implies more frequent replacements. Obviously, this means that the systems would become less reliable. Moreover, the failure of a certain device can cause a failure of a second one. Therefore, the lack of a proactive maintenance program can lead to unexpected costs.

\subsection{Preventive Maintenance}

The main goal of preventive maintenance is to control the degradation of the components in a power system. As result of this, the useful life of these components can be sustained or extended. In order to achieve this, this type of maintenance is based on time schedules. This means that will be pre-defined moments, in which maintenance actions will occur. However, preventive maintenance isn't an optimal maintenance plan. In order to happen, it would imply that the correct components were maintained, at the correct time and with the correct maintenance activities. The mean-time-to-failure (MTTF) or bathtub curve indicates the probability of failure of the components. In preventive maintenance management, machine repairs or rebuilding's are scheduled based on MTTF statistics. The normal result of using MTTF statistics to schedule maintenance is either unnecessary repairs or catastrophic failures. It's true that components require some periodical maintenance actions (lubrification; filter change; etc.), but sometimes this kind of actions can take place, although they can become unnecessary. This is due to the fact that this type of maintenance depends on a schedule and not on the state of degradation of the components. Independently of this, this type of policies will usually result in less components failures. It's possible to make a translation of this fact into cost savings.

\subsection{Predictive Maintenance}

Unlike the preventive maintenance, predictive maintenance is not a time-driven program (Sullivan et al.), (Mobley 2002). Instead, it can be considered as a condition-driven preventive maintenance program. The condition that leads to predictive maintenance actions is related to the state of degradation of the components. The main goals of this type of maintenance are, on the one hand, to take maintenance actions only when necessary and, on the other hand, to prevent the degradation state of a certain component from becoming irreversible.

The predictive maintenance can assume many forms: may be linked to the analysis of the vibration of rotating machinery, or to the monitoring of the infrared image of electrical equipments, or the analysis of oil lubrication. The common premise of predictive maintenance is that regular monitoring of the actual mechanical condition, operating efficiency, and other indicators of the operating condition of the system will provide the data required to ensure the maximum interval between repairs and minimize the number and cost of unscheduled outages. Therefore, the advantages of this kind of maintenance comparatively with preventive policies are of particular interest. First of all, the 
implementation of predictive policies allows to minimize or delete overtime cost and to minimize inventory and order parts. Moreover, it allows to optimize the operation of the equipment, saving energy cost and increasing plant reliability. Despite these advantages, the initial investment is a typical problem.

\section{The sequential Monte Carlo reliability algorithm}

This paper presents the development of several sequential Monte Carlo algorithms. The main differences between them are: the failures rates that can be constant or not and the implementation or not of maintenance programs.

First, the proposed algorithm for a typical sequential Monte Carlo process will be presented. In these types of processes, it is common to use a two-state homogeneous Markov model. Moreover, it's usual to assume that all units are base load units. The use of a constant and independent of time led to a special case known as the exponential distribution. Considering these facts, the state transitions in this Markov model will follow an exponential distribution. The "story of life" of each specific unit is as a source of information. Statistical data can be obtained from it, allowing us to compute the failure and repair rates. Therefore, according to the exponential distribution:

$$
\begin{aligned}
& \lambda=\frac{1}{M T T F} \\
& \mu=\frac{1}{M T T R}
\end{aligned}
$$

where $\lambda$ is the expected failure rate, MTTF is the Mean Time To Failure, $\mu$ is the expected repair rate and MTTR is the Mean Time To Repair.

Taking into account these facts, the probability of finding the unit up can be defined as follows:

$$
P_{u p}=\frac{M T T F}{M T T F+M T T R}
$$

Moreover, the F.O.R or the probability of finding the unit down can be obtained from the following expression:

$$
P_{\text {down }}=\frac{M T T R}{M T T F+M T T R}
$$

It's now time to analyze a different sequential Monte Carlo algorithm. In this new approach, a non constant failure rate will be used. Therefore, some of the characteristics that were presented previously, for a typical Monte Carlo process, must be forgotten. For example, all the simplifications that were introduced by the exponential distribution will no longer take place. In power systems reliability literature, some other distributions are presented and each one of them has their own advantages (Billinton and Allan 1983). The Weibull distribution is an example. This distribution, as well as the gamma and the lognormal, has an interesting property. These distributions have no specific characteristic shape. This means that they can be shaped to represent many distributions or to fit sets of experimental data. It can be achieved by varying its shaping parameters. In (Billinton and Allan 1983), other approach to implement a non constant failure rate is presented. This approach is based on the construction of the cumulative failure distribution $Q(t)$. Through the use of the exponential distribution, $Q(t)$ can be easily defined as:

$$
Q(t)=1-\mathrm{e}^{-\lambda \mathrm{t}}
$$

where $\lambda$ is the failure rate and $t$ is the time variable. The problem is that the exponential distribution uses a constant failure rate. So, in order to develop the cumulative distribution according to a non constant failure rate, the following integral needs to be calculated: 


$$
Q(t)=1-e^{-\int_{0}^{t} \lambda(t) d t}
$$

Furthermore, data about the failure rate variation and about the time intervals will be necessary. This kind of information can be obtained by analyzing statistical data of other similar components. Therefore, through the equation 6 , it is possible to construct a cumulative failure distribution.

After the construction of $Q(t)$, through the use of a non constant failure rate, it is possible to generate the time of operation, of each unit. So, in order to generate the life cycle of each generating unit, the following steps are crucial:

1. Use a pseudo-random number generator to provide an uniformly distributed number in a $[0,1]$ range;

2. The uniformly distributed number is used to intersect the constructed curve. Therefore, $Q(t)=$ uniformly distributed number;

3. The time interval $(t)$ that will result from this intersection will be the new time to failure of a specific component;

4. Repeat the process until all the generating units have their own time to failure.

Another consideration needs to be made about point number 2: $Q(t)$ is a discrete curve. So, a problem must be faced: how it is possible to match a uniformly distributed number with a discrete curve? To overcome this problem, an interpolation process was included in the Monte Carlo simulation. Concluding, this is an approach that allows the introduction of a variable failure rate. This means that the infant mortality and the wear-out regions are included in this method.

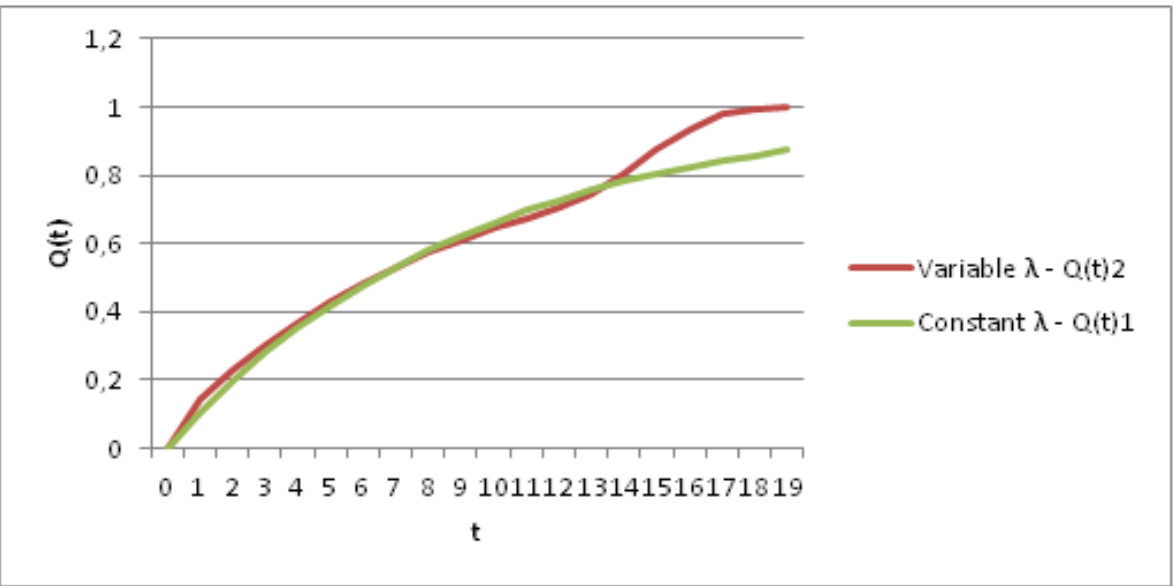

Figure 2: $Q(t)_{1}$ represents a cumulative distribution function developed according to a constant failure rate and, $Q(t)_{2}$ represents the same curve, but developed according to a variable failure rate

The $\lambda$ data used to construct $Q(t)_{2}$ was obtained in (Billinton and Allan 1983). In figure 2, important information can be withdrawn. Two curves are presented: $Q(t)_{1}$ according to a constant failure rate and $Q(t)_{2}$ according to a non constant failure rate. It is crucial to understand why these two curves diverge from $t \geq 14$. The reason behind this fact is the inclusion of the wear-out period in $\lambda$. From $t \geq 14$, the probability of occur a failure in a certain component is higher, when $\lambda$ is non constant. The inclusion of this idea in the Monte Carlo algorithm will have consequences on the reliability indices. Figure 2 will also have interest for the inclusion of maintenance policies. It's is possible to make an analogy between the use of a constant $\lambda$ and an ideal maintenance case. If an ideal maintenance case could exist, the components would not get old. This means that the wear-out region would not exist. The use of a constant $\lambda$ reflects this behavior. Thus, the use a constant failure rate can be compared to an ideal maintenance case. Unfortunately, perfection 
doesn't exist in the maintenance field. On the other hand, the use of a non constant failure rate represents a real situation, in which the components get old with time. The implementation of an effective maintenance plan to this situation would have as consequence the approximation of $Q(t)_{2}$ to $Q(t)_{1}$. In other words, an efficient maintenance procedure can approximate a real situation to a hypothetical situation, in which the components stay forever in their useful life periods.

It is also important to analyze the treatment that will be given to the repair rate. This rate will be treated as in a typical Monte Carlo process. So, the repair rate will be considered constant and, therefore, the exponential distribution will continue to be used.

\subsection{The generation and the load models in Monte Carlo methods}

Beyond the state of the generating units, it is also necessary to know the state of the system load, in order to assess the reliability indices of generating system. Therefore, the data about the generation model isn't enough to evaluate the reliability of a power system. Thus, the evaluation of the reliability indices depends both on the generating units state as the system load state.

In the sequential approach, the load levels are enumerated in chronological order, in which they occur or are expected to occur. This can be on an annual basis or on any other continuous period. This load model can be used to represent the hourly, daily or weekly peaks. In the developed Monte Carlo process, the load model represents the hourly peak, giving 8760 individual peaks.

To sum up, the evaluation of the reliability of a generating system depends on the verification, for each state of the generating system, if the available capacity is enough or not to ensure the load model requirements.

\subsection{Inclusion of the maintenance techniques in the algorithm}

In section 3.1. of this paper, the first maintenance technique was presented: the reactive maintenance. Although the reactive policy is associated with the term "maintenance", there are no maintenance actions in this type of program. In other words, the repairs of the generating units only occur after they fail. For this reason, to add this kind of maintenance on the typical Monte Carlo process, no changes are needed. On the other hand, the other two types of maintenance that will be discussed are based on active measures. These actions are taken during the operation of the generating units. As a consequence, new code parcels need to be developed, in order to introduce this new behavior on a typical Monte Carlo process. The inclusion of these two methods pretends to compare the time-driven programs with the condition-driven programs.

The main goal of this research is to evaluate and analyze the positive effects of these maintenance programs. Therefore, the units won't be removed from service when maintenance actions are taken. So, the maintenance actions will not affect the capacity available for service.

\subsubsection{Preventive Maintenance inclusion}

A well-structured maintenance plan, in which the goals are clearly defined, can lead to major improvements in the reliability of power systems. The developed Monte Carlo simulations will prove that these improvements are real.

In the theoretical plan, the maintenance actions have the main goal of extend the useful life period of the generating units. In other words, these policies pretend to delay the entry of these units in the wear-out period. 
In the simulation plan, some changes needs to be incorporated, in order to add the maintenance policies. First, in the preventive maintenance, it is necessary to establish the maintenance schedule. This particularity needs to be implemented in the Monte Carlo simulation. In order to understand the process of implementing this maintenance policy, it is necessary to examine the steps presented in section 4 that describe the generation of the life cycle of each generating unit. Through this algorithm, it is easy to realize that the whole process of simulation depends on the failure rate $\lambda$. The construction of the cumulative function $Q(t)$ and the generation of the time of operation of each component, depend on $\lambda$. It is, therefore, by manipulating the failure rates, that the expected effect of maintenance can be incorporated. Thus, whenever a new operation time is generated, it is necessary to check the maintenance schedule. If the lifetime of a given component is equal to one of the scheduled maintenances, maintenance actions will take place. This will involve changes in the failure rate of this component. The fact that a given component receives periodic maintenance actions, will lead to an extension of his useful life period. In figure 3, a comparison between the failure rates of two components with the same characteristics is made: one of them has periodical maintenance actions and the other one follows the reactive maintenance methodology.

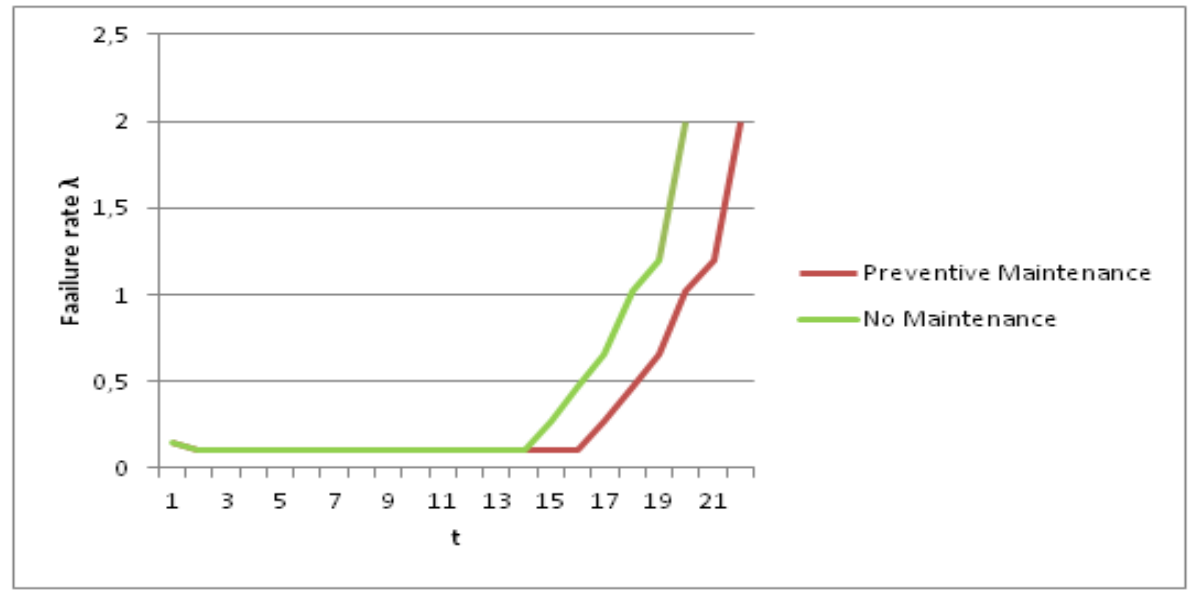

Figure 3: Effect of preventive maintenance actions upon the failure rate $\lambda$ : the extension of the useful life period Considering the algorithm presented in section 4 , it is easy to realize that the changes produced on $\lambda$ have as consequence the recalculation of the $Q(t)$ curve. Only through this new curve, the generation of the times of operation of each unit will include the effect of maintenance.

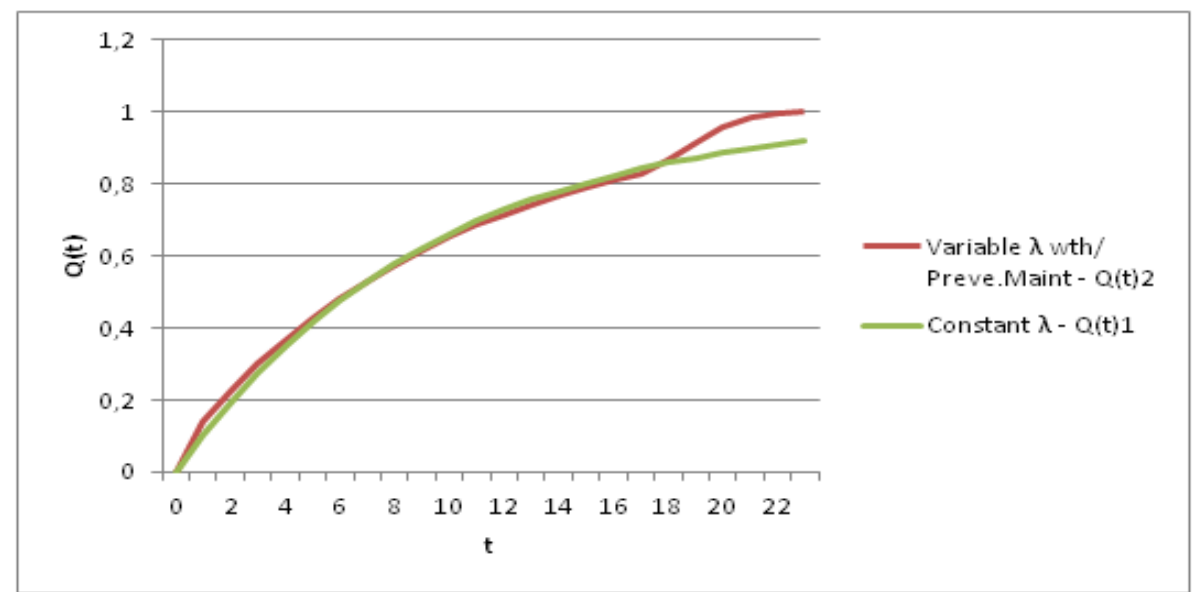

Figure 4: Effect of preventive maintenance actions upon $\mathrm{Q}(\mathrm{t})$ curve: $Q(t)_{2}$ diverge from $Q(t)_{1}$ in a later stage of the generating unit life 
Figure 4 allows to observe something very important. The periodical maintenance actions had the expected effect on the cumulative curve: $Q(t)_{1}$ is now closer to $Q(t)_{2}$. In other words, a real situation (variable $\lambda$ ) is now closer to a hypothetical situation (constant $\lambda$ ) known for the ideal maintenance case. Therefore, the generating units enter on the wearout period, in a later stage of their lives $(t \geq 18)$. Obviously, this fact will have clear effects on the reliability indices. The extension of the useful life periods of the generating units, will lead to less failures during their lives. Therefore, in the results section, the variations on the reliability indices will correspond to this effect.

It is, still, important to mention one more aspect. The magnitude of the extension of the useful life periods gives a measure of the effectiveness of these maintenance plans. The preventive maintenance is not the more effective maintenance policy, since it does not evaluate the current state of components. For this reason, the increase of the useful life period, in this type of maintenance, will correspond to only one period of time, at each scheduled maintenance. After introducing the preventive maintenance basic ideas, now the steps of this methodology are explained in detail. This algorithm is based on a set of different approaches that can be found in (Billinton and Allan 1983) and (Miranda, 2005).

1. I nitialize the reliability characteristics of the generating units:

o $\lambda$ - failure rates;

o MTTR;

o Number of uni ts;

o Capacity of each uni $t$.

2. I nitialize the power systemload curve.

3. I ni tial ize the random number gener at or and ot her variables as:

o Maxi mum number of years of the si mul ati on process;

o $\beta$ threshol d: $\beta_{\max }=5 \%$.

4. For each generating unit, gener ate a ti me of operation according to the al gor ithm presented i $n$ sect $i$ on 3. 2 .

DO

5. I dentify the l owest si mul at ed time. The corresponding component will be call ed F.

6. Update the system load for this particul ar moment.

7. State eval uation. According to the current state of each component, the I oad curtai I ment is cal cul ated.

8. Update the rel i abil i ty i ndi ces accumul at ors.

9. Si mul at e a new operating or repai $r$ ti me to the component $F$. The type of si mulated ti me depends on the previ ous state of the component F.

9. 1. If component $F$ was in operat i onal node, a repai $r$ time will be gener at ed. As i t was st udi ed bef ore, the gener ati on of a repai $r$ ti me will foll ow an exponential di stribution.

9. 2. If component $F$ was in repai $r$ mode, an operat $i$ on ti me will be gener at ed, according to the al gor $i$ thm present ed i n sect $i$ on 4 .

9. 2. 1. If the I i fet i me of component $F$ is equal to one of the pre-defined moments of the scheduled maintenances, $\lambda$ must be updat ed.

9. 2. 2. Update the $Q(t)$ curve.

9. Update the component $S \mathrm{~F}$ st ate and its I i fet i me.

10. Eval uate the I if et i me of the system Tests if the system has al ready compl et ed one year and make the necessary updates, i ncl uding the update of the coefficient of variation $\beta$.

Until the maxi mumgeneration criteria. $\beta_{\max }=\mathbf{5} \%$.

11. Compute the fol I owing rel i abil i ty i ndi ces:

o Loss of Load Probabi I ity (LOLP) ;

- Expect ed Power Not Suppl i ed (EPNS);

o Loss of Load Dur at i on ( LOLD) .

12. Compute the probability distributions of Loss of Load Expectation (LOLE), Expect ed Energy Not Suppl i ed (EENS) and Loss of Load Frequency ( LOLF). 


\subsubsection{Predictive Maintenance inclusion}

Predictive and preventive maintenance programs have the same main goal: to extend the useful life period of the generating units. The difference between them lies on the moments in which the maintenance actions occur. On the one hand, preventive maintenance actions are performed according to a maintenance schedule. On the other hand, predictive maintenance operations are performed, when a given component enters its last stage of life: the wear-out period. The implementation of the predictive maintenance will also lead to some changes on the Monte Carlo algorithm. In first place, instead of defining the maintenance schedule, it is necessary to define the moment when the failure rate $\lambda$, of each generating unit, starts to increase (wear-out period). Therefore, whenever a new time of operation is generated for a given unit, it is necessary to check its lifetime. In other words, it is necessary to verify if this unit already entered in its wear-out period. Maintenance actions will be the consequence to a positive answer to this question. Thanks to these maintenance actions, the useful life of the generating units will be extended. Therefore, the main goal of the predictive maintenance can be defined as follows: whenever the failure rate $\lambda$, of a given unit starts to increase, bring this $\lambda$, through the maintenance actions, to its useful life period. The consequences of the predictive maintenance for the failure rate $\lambda$ can be observed in figure 5:

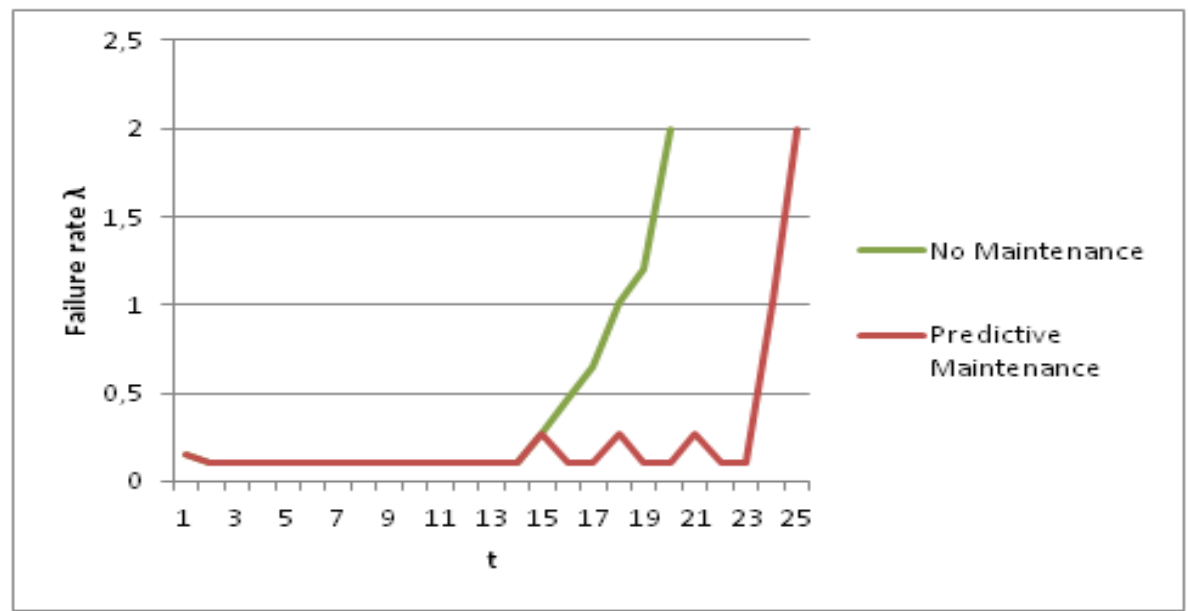

Figure 5: Effect of predictive maintenance actions upon the failure rate $\lambda$ : the extension of the useful life period As it was expected, whenever $\lambda$ increases, maintenance actions are taken in order to bring $\lambda$ to its useful life period. The following step is the recalculation of the $Q(t)$ curve. Figure 6 show the results that were obtained.

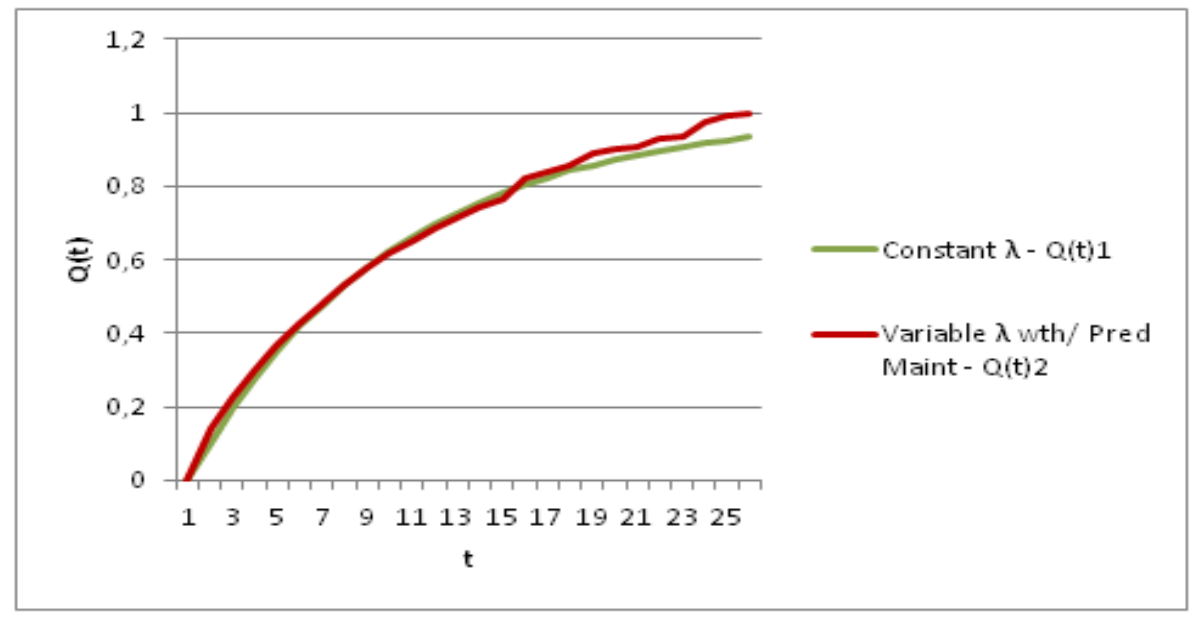

Figure 6: Effect of predictive maintenance actions upon the $\mathrm{Q}(\mathrm{t})$ curve: $Q(t)_{2}$ diverge from $Q(t)_{1}$ in a later stage of the generating unit life 
The main idea to withdraw from the previous image is the following one: with a predictive maintenance process, the generating units enter on their wear-out periods in a later stage of their lives. Once more, this type of maintenance also had the expected effect on $Q(t)$ : a real situation, in which the $\lambda$ is variable, is now closer to a hypothetical situation, in which $\lambda$ is not constant. Considering the above points, is expected that the predictive maintenance will lead to better reliability indices than the preventive maintenance. It is still important to discuss the subject of the magnitude of the useful life period extension. It was possible to realize, in the literature, that the predictive maintenance constitutes a more effective plan. For this reason, the increase of the useful life period, in this type of maintenance, will correspond to two periods of time, at each maintenance procedure.

The algorithm presented in this section for the preventive maintenance is very similar to the algorithm that will include the predictive policies. The only difference between these two algorithms is related to the maintenance moments. Therefore, the step 9.2.1 of the algorithm can be defined as follows:

9. 2. 1. If the I if et i me of component $F$ is equal or superior to the moment, in whi ch $\lambda$ starts to i ncrease, mai nt enance act i ons wi 11 be performed. $\lambda$ is updated;

\section{Results and discussions}

In order to evaluate the adequacy of the generating capacity, the IEEE RTS-79 (IEEE 1979) was the chosen power system. Therefore, the proposed methodologies will be tested in this power system. The need of a standardized power system to test and compare results from different reliability approaches, led to the development of this and other standardized systems. These power systems are characterized for having a standardized database. In IEEE RTS-79, a lot of information and data are described, as for example, transmission network data. In the addressed problem, only two types of data are needed: the generation data and the load model.

\subsection{Evaluation of the Monte Carlo performance with a constant failure rate $\lambda$}

The development of a typical Monte Carlo algorithm was the first step taken in this work. This algorithm is based on one particularity: the failure rates $\lambda$ of the generating units are constant and, therefore, an exponential distribution is followed. The reliability indices that were obtained can be seen in the following table:

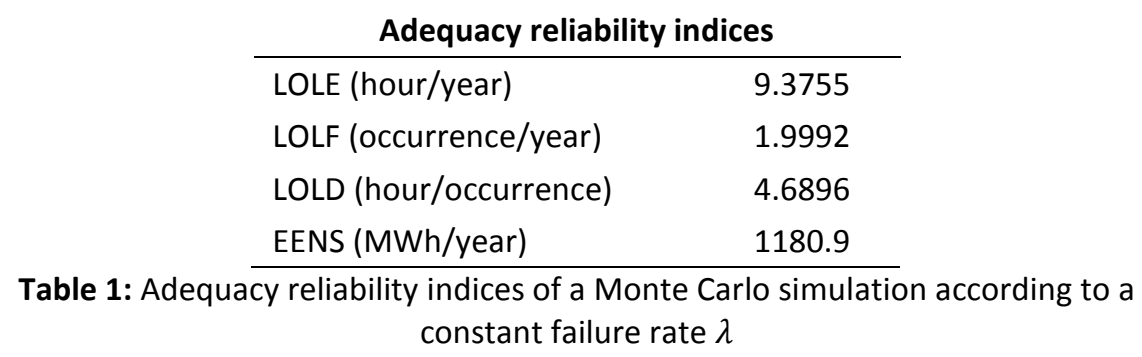

These results are very important because they allow to validate the followed methodology. However, these results will have more interest, when compared to the results of the other methodologies.

\subsection{Evaluation of the Monte Carlo performance with a non constant failure rate $\lambda$}

Theoretically, the difference between this new approach and the typical method is very simple: the constant failure rate $\boldsymbol{\lambda}$ is replaced for a variable failure rate $\boldsymbol{\lambda}$. In the other hand, in practice, the changes produced in the Monte Carlo algorithm are several, as it was already explained in the previous section. Figure 7 presents the adopted "bathtub curve" of the nuclear units. 
Figure 7 shows that, in this new approach, the infant mortality and wear-out periods are included.

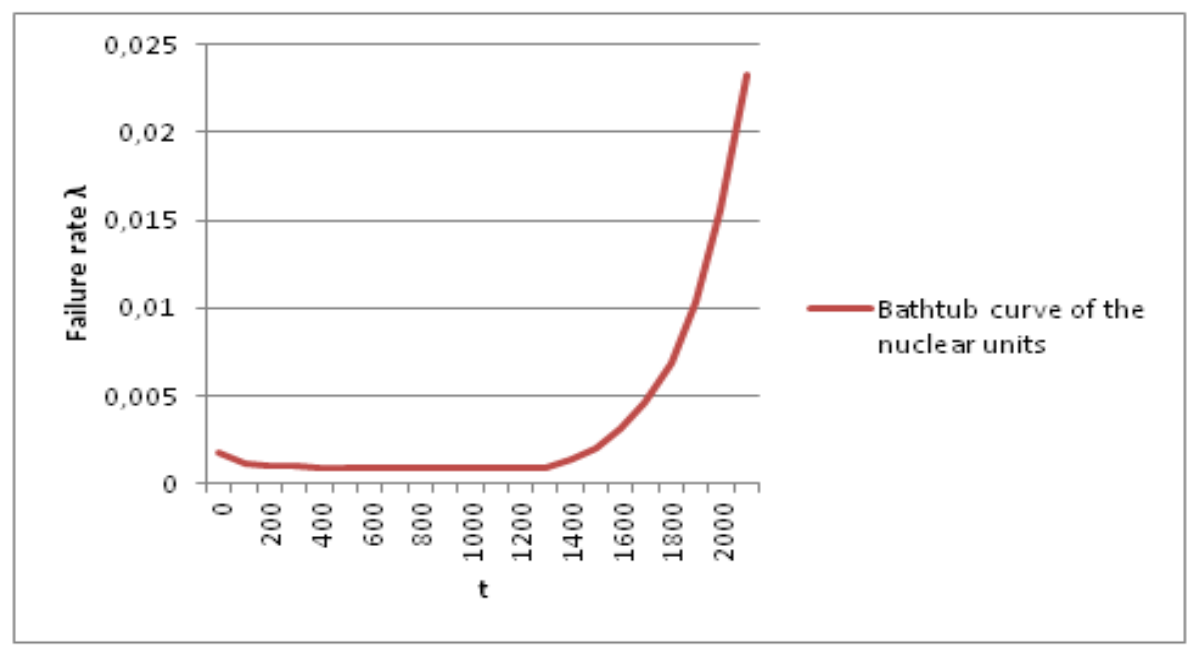

Figure 7: Bathtub curve that will be implemented in the Monte Carlo algorithm, instead of a constant failure rate. The data of this curve can be inspected in (Silva 2014)

Therefore, a real situation is presented, in which the nuclear units have debugging problems at the beginning of their lives and, in which the degradation process with the elapse of time is clearly visible. According to the algorithm presented, the changes produced on the failure rate $\lambda$ have as consequence the recalculation of the $Q(t)$ curve. The new $Q(t)$ curve is presented in figure 8:

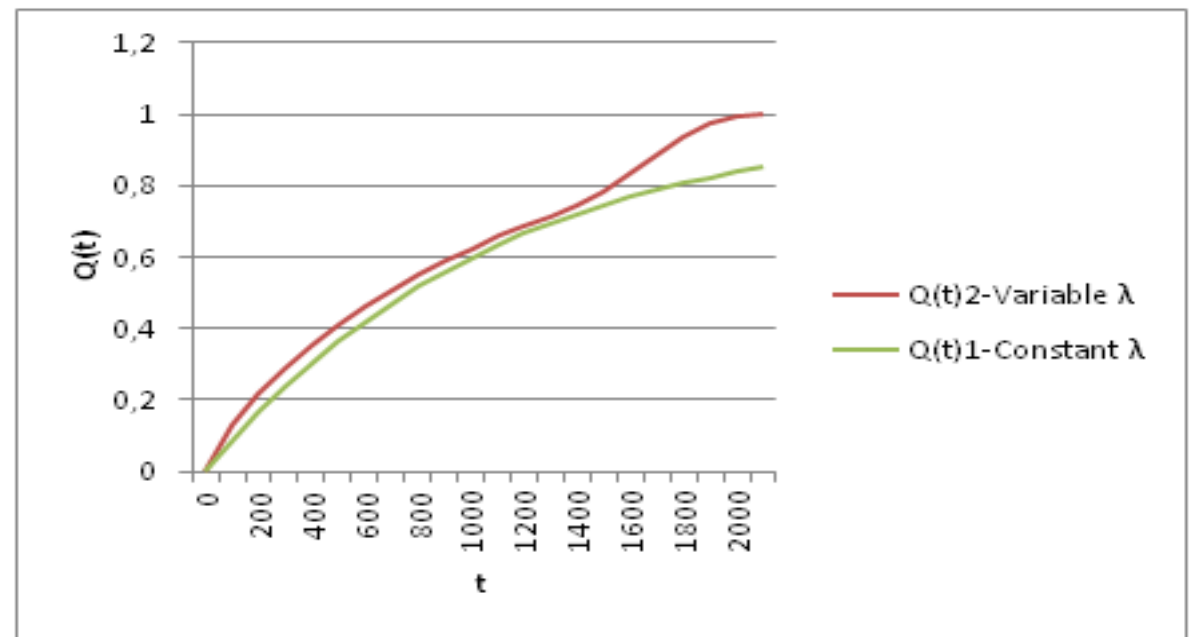

Figure 8: Two $Q(t)$ curves for the nuclear units: $Q(t)_{1}$ according to a constant $\lambda$ and $Q(t)_{2}$ according to the bathtub curve presented in figure 7

Figure 8 shows a comparison between two $Q(t)$ curves: one is constructed according to a constant failure rate $\lambda$ and the other one is developed according to a variable failure rate $\lambda$. The main feature to withdraw from figure 8 is the divergence shown by the curves from $t \geq$ $1400 h$. The explanation of this divergence is the inclusion of the wear-out period in $Q(t)_{2}$. From $\boldsymbol{t} \geq \mathbf{1 4 0 0 h}$, the probability of failure, $\boldsymbol{Q}(\boldsymbol{t})$, of this generating unit is much higher, when the failure rate is not constant. This is a normal consequence of the introduction of the wear-out period, which is characterized for an increasing failure rate. It is also important to mention that this is a comparison between a reactive maintenance plan and an ideal maintenance case. The reactive maintenance is a type of maintenance program, in which maintenance action are not taken. In other words, the generating units are repaired only in case of failure. Therefore, the use of a non constant failure rate can be considered a case of 
reactive maintenance. The next goal, in this work, is to approach the two curves presented in figure 8 , through the implementation of maintenance policies.

\begin{tabular}{lr}
\multicolumn{2}{c}{ Adequacy reliability indices } \\
\hline LOLE (hour/year) & 11.1961 \\
LOLF (occurrence/year) & 2.3256 \\
LOLD (hour/occurrence) & 4.8144 \\
EENS (MWh/year) & 1486.1 \\
\hline
\end{tabular}

Table 2: Adequacy reliability indices of a Monte Carlo simulation according to a non constant failure rate $\lambda$

These results were obtained according to a coefficient of variation $\beta=5 \%$. As it was expected, these reliability indices are higher, when compared to the ones obtained for a constant failure rate $\lambda$. The inclusion of the degradation process led to an increase in the number of failures of the generating units. Therefore, the increase of the reliability indices illustrates this consequence. Moreover, these indices are more realistic, since they are the result of a real situation.

\subsection{Evaluation of the Monte Carlo performance with a preventive maintenance program}

The first task was the definition of the maintenance schedule in order to implement it in the developed Monte Carlo algorithm. This definition depends on some characteristics of the generating units, as for example, the MTTF. Furthermore, each generating unit has their own characteristics. For this reason, it was decided to apply the maintenance program only to one of the types of the generating units. By choosing units with higher capacity, the effect of maintenance upon the reliability indices is clearer. Therefore, the nuclear units were the chosen ones. According to the characteristics of the nuclear units, which can be inspected in (Silva 2014), the maintenance actions will occur at each $400 \mathrm{~h}$. Thanks to these maintenance actions, the useful life of the nuclear units will be extended, as it is possible to observe in figure 9:

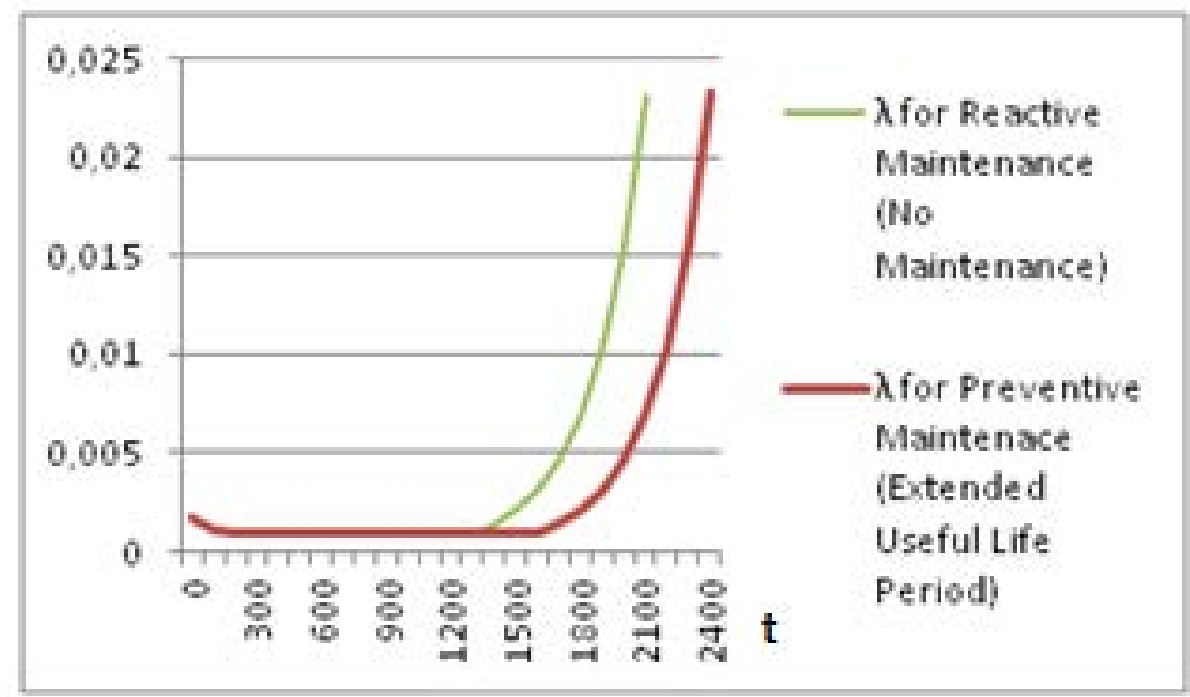

Figure 9: Extended useful life period of the nuclear unit, after a preventive maintenance procedure

Then, the $Q(t)$ curve needs to be recalculated. Therefore, the new $Q(t)$ curve will include the effect caused by the useful life extension. 


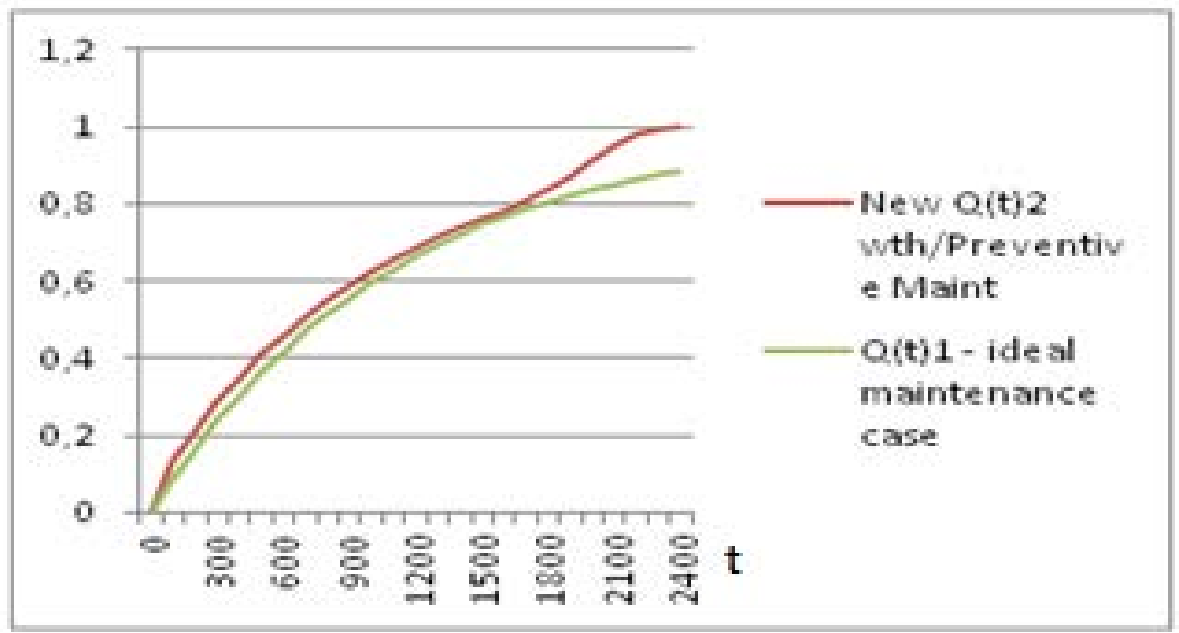

Figure 10: The preventive maintenance plan delays the entrance on the wear-out period

In figure 10, the effect of preventive maintenance is clearly observable. $Q(t)_{2}$ represents the cumulative curve of the nuclear units including the scheduled maintenances. By comparing this figure with figure 8 , main points to withdraw are the following:

- When maintenance actions are not taken, these generating units enter on their wearout periods after $t=1400 h$;

- When preventive maintenance is applied, these generating units enter on their wearout periods after $t=1700 \mathrm{~h}$.

So, the changes produced on the $Q(t)$ curve are beneficial for the reliability of the power systems. By introducing the maintenance actions, the failure rate $\lambda$ of the nuclear units starts to increase in a later stage of their lives. Ultimately, the reliability indices can measure the effects produced by the maintenance policies.

\begin{tabular}{lr}
\multicolumn{2}{c}{ Adequacy reliability indices } \\
\hline LOLE (hour/year) & 10.8822 \\
LOLF (occurrence/year) & 2.2621 \\
LOLD (hour/occurrence) & 4.8107 \\
EENS (MWh/year) & 1406.2 \\
\hline
\end{tabular}

Table 3: Adequacy reliability indices of a Monte Carlo simulation, after the implementation of the preventive maintenance

After analyzing these results, it is possible to conclude that the preventive maintenance plan had the expected effect: the reliability indices are, now, lower when compared to the ones presented for the reactive maintenance.

\subsection{Evaluation of the Monte Carlo performance with a predictive maintenance program}

The first step to take is to identify the moment when the generating units enter on their wear-out periods. This moment is different for each generating unit and depends on their failure rates $\lambda$. So, in order to establish a comparison between the two studied maintenance types, the predictive maintenance will be applied on the nuclear units. As it was seen before, after $t=1400 h, Q(t)_{1}$ and $Q(t)_{2}$ start to diverge. This is exactly the moment when the nuclear units enter on their wear-out period. Therefore, always that these units enter on their wear-out period, maintenance actions will be taken. In figure 11, the effect of predictive maintenance upon the failure rate $\lambda$ of the nuclear units can be observed: 


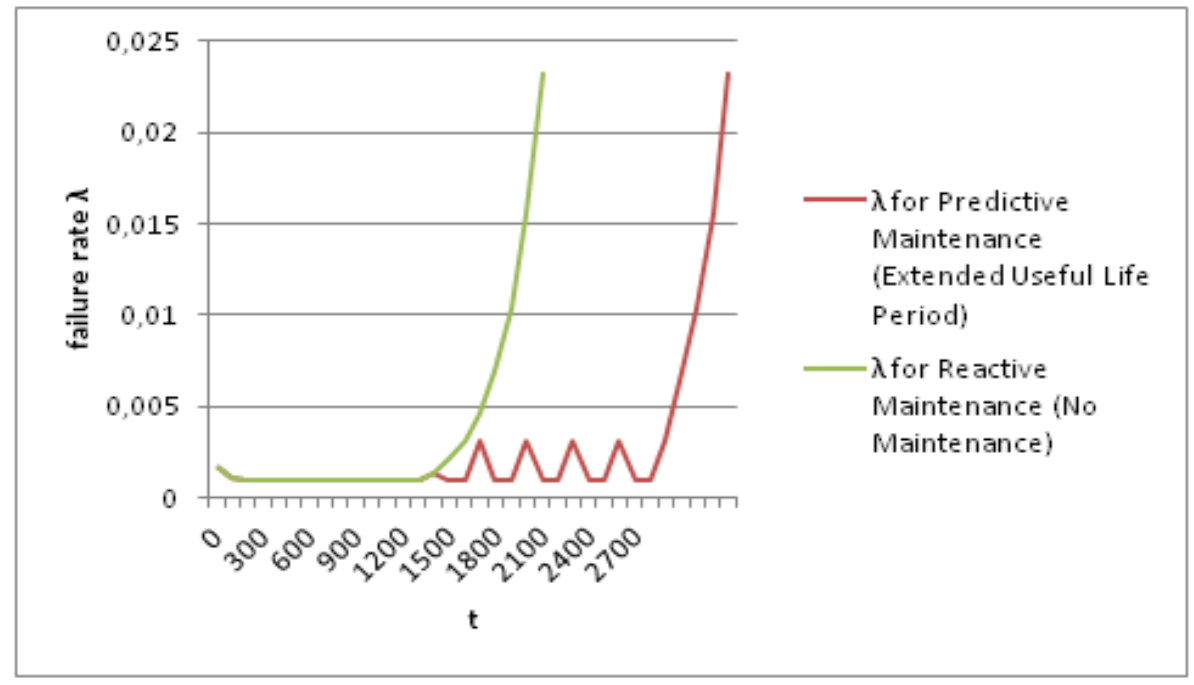

Figure 11: Extended useful life period of the nuclear unit, after a predictive maintenance procedure

The ups/downs of the failure rate $\lambda$ in figure 11, reflect the type of maintenance that was applied. Whenever $\lambda$ increases, maintenance actions are taken in order to bring $\lambda$ to its useful life period. Thus, the existence of unnecessary maintenance actions is avoided. The produced changes on $\lambda$ led to the reconstruction of the $Q(t)$ curve.

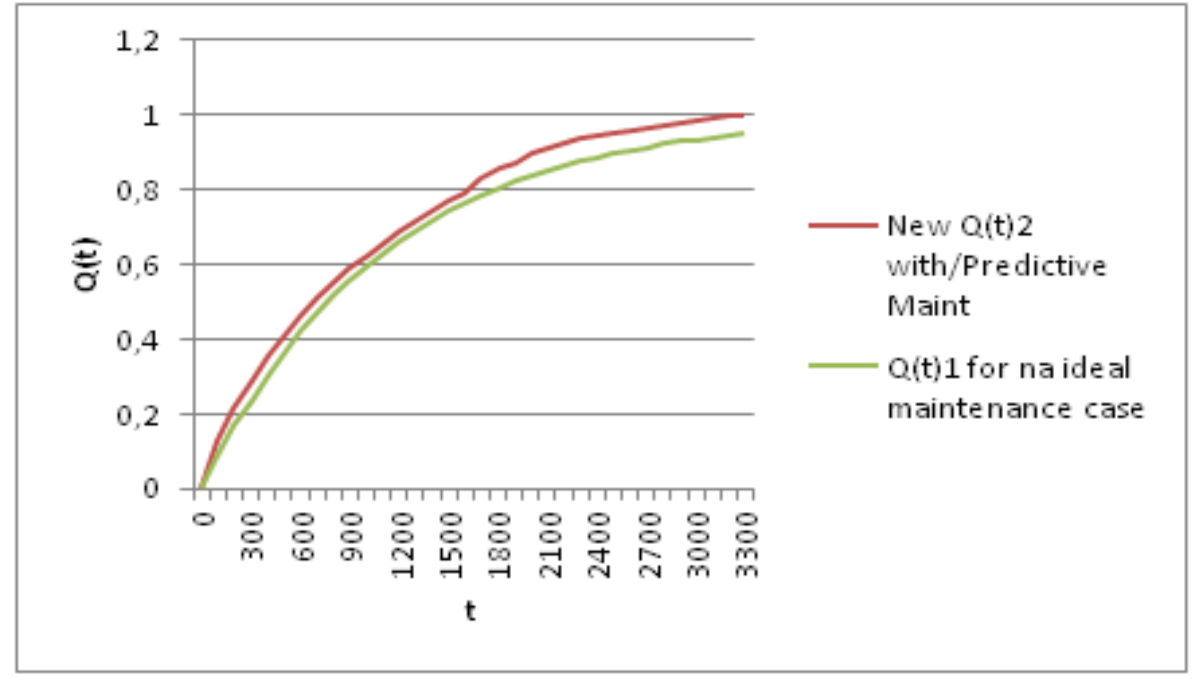

Figure 12: Effect of predictive maintenance actions upon $Q(t)$ curve, delaying the entrance on the wear-out period

As it was expected, thanks to the predictive maintenance plan, the nuclear units enter on their wear-out period in a later stage of their lives (after $t=2900 \mathrm{~h}$ ). By comparing figure 12 with figures 10 and 8 (preventive and reactive maintenance), the advantages of predictive maintenance are clearly recognizable. In fact, the main conclusions to withdraw from this comparison are the following:

- With a predictive maintenance plan, the nuclear units enter on their wear-out period $1200 \mathrm{~h}$ later, when compared with the preventive policies.

- With a predictive maintenance plan, the nuclear units enter on their wear-out period $1500 h$ later, when compared with the reactive policies.

Therefore, a predictive maintenance leads to a bigger extension of the useful life period of the nuclear units policy. For last, but not least, the reliability indices for a predictive maintenance are presented: 


\begin{tabular}{lr}
\multicolumn{2}{c}{ Adequacy reliability indices } \\
\hline LOLE (hour/year) & 10.4864 \\
LOLF (occurrence/year) & 2.1897 \\
LOLD (hour/occurrence) & 4.7892 \\
EENS (MWh/year) & 1379.1 \\
\hline
\end{tabular}

Table 4: Adequacy reliability indices of a Monte Carlo simulation, after the implementation of the predictive maintenance

Table 4 shows that the reliability indices decrease regarding to the ones obtained for a preventive maintenance plan. This was already expected, since the predictive maintenance is based on a continuous inspection of the generating units state.

\section{Conclusions}

Through the developed work, several important conclusions can be withdrawn. First, it was proved that the inclusion of the real variation of the failure rate $\lambda$ has a significant impact upon the reliability indices. Although this new approach is more complex, it allows to include the impact of the natural process of degradation of electrical components. Therefore, with this new approach, it's possible to understand the moments when maintenance actions should occur. Through the results obtained, it was possible to figure out that the inclusion of the maintenance policies allowed to extend the useful life period of the nuclear units. Furthermore, the predictive maintenance was responsible for a higher increase of the useful life period. This was already expected since the predictive maintenance is based on a continuous inspection of the generating units state. To sum up, it was proved that a more realistic Monte Carlo algorithm can be developed in order to evaluate the reliability of power systems.

\section{References}

IEEE Reliability Test System Task Force of the Application of Probability Methods Subcommittee. 1979. "IEEE reliability test system", IEEE on Power Apparatus and Systems, 2047-2054.

Miranda, Vladimiro. 2005. Reliability of Power Systems, Universidade Federal do Pará.

Billiton, Roy, and Ron Allan. 1996. Reliability Evaluation of Power Systems. New York: Plenum Press.

Billiton, Roy, and Wenyuan Li. 1994. Reliability Assessment of Electric Power Systems using Monte Carlo Methods. New York: Plenum Press.

Sankarakrishnan, A., and Roy Billinton. 1995. "Sequential Monte Carlo Simulation for Composite Power System Reliability Analysis with Time Varying Loads", IEEE Trans. Power Systems, vol.10, no3.

Billiton, Roy, and Ron Allan. 1983. Reliability Evaluation of Engineering Systems: Concepts and Techniques. New York: Plenum Press.

Miranda, Vladimiro. 2012. Monte Carlo Cronológico ou Sequencial. Faculdade de Engenharia da Universidade do Porto.

Sullivan, G., R. Pugh, A. Melendez and D. Hunt. 2010. Operations \& Maintenance Best Practices. U.S. Department of Energy.

Mobley, K. 2002. An Intoduction to Predictive Maintenance. Butterworth- Heinemann 2으dition.

Silva, João. 2014. "Definition of Maintenance Policies in Power Systems", Msc diss., Faculty of Engineering of Oporto. 